\title{
Critical Consciousness in Nursing Education / Conscience critique en formation infirmière
}

Jacinthe I. Pepin

Université de Montréal, jacinthe.pepin@umontreal.ca

Florence Myrick

amyrick@ualberta.ca

Follow this and additional works at: https://qane-afı.casn.ca/journal

Part of the Nursing Commons, and the Scholarship of Teaching and Learning Commons

\section{Recommended Citation}

Pepin, Jacinthe I. and Myrick, Florence (2021) "Critical Consciousness in Nursing Education I Conscience critique en formation infirmière," Quality Advancement in Nursing Education - Avancées en formation infirmière: Vol. 7: Iss. 2, Article 1.

DOI: https://doi.org/10.17483/2368-6669.1327

This Editorial is brought to you for free and open access by Quality Advancement in Nursing Education - Avancées en formation infirmière. It has been accepted for inclusion in Quality Advancement in Nursing Education - Avancées en formation infirmière by an authorized editor of Quality Advancement in Nursing Education - Avancées en formation infirmière. 


\section{Critical Consciousness in Nursing Education}

In light of the serious events related to today's care of Indigenous People in the Canadian health care system, the death of Ms. Joyce Echaquan, Atikamekw, being one, many have wondered during the past year why health professionals have been so oblivious to the various forms of racism and discrimination. Health professionals have been taught to engage in person- and family-centred care and partnership relations with patients, concepts further emphasized particularly within postgraduate programs that foster the advancement of their critical thinking, advocacy, and culturally safe care, etc. (Canadian Association of Schools of Nursing [CASN], 2015; Frank et al., 2015).

This complex issue warrants in-depth scrutiny and would certainly require more than one perspective and more than a few lines to be fully understood, given justice, and resolution. We are, however, opening a conversation on "critical consciousness" in nursing education, distinct from the "critical thinking" concept, that is currently described as a decontextualized generic set of cognitive skills and affective dispositions, or habits of the mind (Lavoie \& Deschênes, submitted; Monteiro et al., 2020). Critical thinking facilitates, for example, discernment of relevant knowledge for its use in practice but seems to have lost its socio-political dimension.

The critical consciousness concept, also having both cognitive and affective components, is described as a "reflective reading of the world, with focused attention upon societally embedded inequities" (Halman et al., 2017, p. 13). Proposed by the pedagogist Freire to transform education in Brazil, in the 1970's, this concept calls the learners to act as change agents. In their literature review on the use of critical consciousness to inform the health professions' education, Halman et al. (2017) found that 20/30 articles were written by authors in the nursing profession, that 14/30 articles were published in the last five years, and that the majority of articles were conceptual or theoretical in nature, indicating that this approach might rarely influence educational practices.

Nursing schools are definitively engaged in contributing to health equity for Indigenous Peoples as it was declared by the Canadian Association of Schools of Nursing in June 2021 (CASN, 2021). Globally, however, nursing education might still have to commit to what has been described as education of health professionals for the twenty-first century, for a significant contribution to health equity, and to countering racism and discrimination. Indeed, it was proposed, by an international commission, that professional education be transformative and interdependent for the purpose of fostering the development of "change agents with the status, authority, and ability to promote enlightened transformation in society", health equity for marginalized being one such needed transformation (Frenk et al., 2010, p. 1946).

Thus, to improve care for Indigenous Peoples and for other marginalized people, shall nursing education focus on supporting the learners' critical consciousness together with clinical judgement in nursing (Beauchamp et al.; Lavoie et al. in this edition of Quality Advancement in Nursing Education [QANE]), ethics in nursing, and other salient outcomes? Notably, O'FlynnMagee et al. (in this edition of QANE) founded their Cognitive Rehearsal to Address Bullying (CRAB) workshop on Freire's notions of power, oppression, and human agency to foster change through participatory learning activities. Attention on how best to support the development of critical consciousness in learners is also of the utmost importance.

Examples could be drawn from the San'yas Indigenous cultural safety educational intervention which is aptly becoming more widely known. This Indigenous-led, policy-driven, and system-level educational intervention as pointed out by Browne et al. (2021, p. 1) "aims to foster 
health equity and mitigate the intersecting forms of interpersonal and structural racism experienced by Indigenous people in the health care system in Canada." Characteristics of the program point to specific teaching by and about Indigenous People, an approach that aims at transforming thoughts and actions, and a system-level intervention, inasmuch as education alone cannot sustain a major change without organizational and systemic policies (Browne et al., 2021).

To continue this conversation on health and care equity for Indigenous people, we are honored to welcome Dr. Lisa B. Bearskin, Dr. Andrea Kennedy, and Dr. Wanda Phillips-Beck, as Guest Editors for a future special edition of QANE. Stay tuned!

Jacinthe Pepin, RN PhD, Co-Editor-in-Chief - Corédactrice en chef

Florence Myrick, RN PhD, Co-Editor-in-Chief - Corédactrice en chef

\section{References}

Browne, A., Varcoe, C., \& Ward, C. (2021). San'yas Indigenous Cultural Safety Training as an educational intervention: Promoting anti-racism and equity in health systems, policies, and practices. The International Indigenous Policy Journal, 12(3). https://doi.org/10.18584/iipj.2021.12.3.8204

Canadian Association of Schools of Nursing. (2015). National Nursing Education Framework. Final report. Author. https://www.casn.ca/wp-content/uploads/2014/12/FramworkFINAL-SB-Nov-30-20151.pdf

Canadian Association of Schools of Nursing. (2021, June 29). Canadian Association of Schools of Nursing Statement of Commitment Anti-Indigenous Racism. https://www.casn.ca/wpcontent/uploads/2021/07/CASN-Anti-Indigenous-Statement-of-Commitment-EN.pdf

Frank, J. R., Snell, L., \& Sherbino, J. (Eds.) (2015). CanMEDS 2015 Physician Competency Framework. Royal College of Physicians and Surgeons of Canada. http://canmeds.royalcollege.ca/uploads/en/framework/CanMEDS\%202015\%20Framewor k_EN_Reduced.pdf

Frenk, J., Chen, L., Bhutta, Z. A., Cohen, J., Crisp, N., Evans, T., Fineberg, H., Garcia, P., Yang, K., Kelley, P., Kistnasamy, B., Meleis, A., Naylor, D., Pablos-Mendez, A., Reddy, S., Scrimshaw, S., Sepulveda, J., Serwadda, D., \& Zurayk, H. (2010). Health professionals for a new century: Transforming education to strengthen health systems in an interdependent world. The Lancet, 376(9756), 1923-1958. https://doi.org/10.1016/s0140$\underline{6736(10) 61854-5}$

Lavoie, P., \& Deschênes, M-F. (in press). Fostering the development of clinical judgment and reasoning in the clinical setting. In S. Melrose \& B. Perry (Eds.), Clinical Teaching in Canadian Nursing. Canadian Association of Schools of Nursing.

Monteiro, S., Sherbino, J., Sibbald, M., \& Norman, G. (2020). Critical thinking, biases and dual processing: The enduring myth of generalisable skills. Medical Education, 54(1), 66-73. https://doi.org/10.1111/medu.13872 


\section{Conscience critique en formation infirmière}

Au regard de graves événements survenus lors de soins reçus par des personnes des Premiers Peuples dans le système de santé canadien, parmi eux le décès de Mme Joyce Echaquan, Atikamekw, plusieurs se sont demandé au cours de la dernière année, comment les professionnels de la santé ont-ils pu être si inconscients des diverses formes de racisme et de discrimination. Les professionnels de la santé ont appris à donner des soins centrés sur la personne et la famille et à établir des relations partenariales avec les patients, des concepts soulignés à grands traits dans des programmes universitaires qui soutiennent le développement du jugement clinique, de l'advocacy, de soins culturellement sécuritaires et autres (Association canadienne des écoles de sciences infirmières [ACESI], 2015; Frank et al., 2015).

Cet enjeu complexe requiert un examen en profondeur à partir de plus d'une perspective et nécessite certainement plus de quelques lignes sur le sujet afin d'en comprendre les contours, de lui rendre justice et de tenter de le cerner. Cependant, nous ouvrons une conversation sur la « conscience critique » en formation infirmière, un concept distinct de la «pensée critique »; ce dernier est actuellement décrit comme un ensemble générique d'habiletés cognitives et de dispositions affectives, à utiliser quel que soit le contexte, ou comme des habitudes de pensée (Lavoie \& Deschenes, sous presse; Monteiro et al., 2020). La pensée critique soutient, par exemple, le choix judicieux de connaissances pertinentes à intégrer dans la pratique infirmière mais semble avoir perdu sa dimension sociopolitique.

Le concept de conscience critique, comportant également des composantes cognitives et affectives, est plutôt décrit comme « une lecture réflexive du monde avec une attention particulière portée aux iniquités sociales qui y sont imbriquées » (traduction libre, Halman et al., 2017, p. 13). Proposé par le pédagogue théoricien Freire pour transformer l'éducation au Brésil dans les années 1970, ce concept interpelle les apprenants à agir comme agents de changement. Dans le cadre de leur recension des écrits sur l'utilisation de la conscience critique comme fondement pour la formation des professionnels de la santé, Halman et al., (2017) ont noté que 20/30 des articles retenus étaient rédigés par des auteurs de la profession infirmière, que 14/30 articles avaient été publiés au cours des cinq années précédant la recension et que la majorité des articles étaient de nature conceptuelle ou théorique, indiquant que ce fondement guidait encore peu les pratiques de formation.

Les écoles de sciences infirmières sont résolument engagées à contribuer à l'équité en santé des personnes des Premiers Peuples, tel que l'Association canadienne des écoles de sciences infirmières l'a déclaré en juin 2021 (ACESI, 2021). Cependant, de manière générale, la formation en sciences infirmières pourrait encore devoir s'engager vers une formation conçue pour les professionnels de la santé du $21^{\text {ième }}$ siècle, afin de contribuer de manière significative à promouvoir l'équité en santé et à contrer le racisme et la discrimination. En effet, une commission internationale a proposé que la formation des professionnels de la santé devait être transformée et interdépendante dans le but de soutenir le développement « d'agents de changement ayant le statut, l'autorité et la capacité de promouvoir une transformation éclairée dans la société » (traduction libre, Frenk et al., 2010, p. 1946); l'équité en santé pour les personnes marginalisées représente une des transformations essentielles.

Ainsi, dans le but d'améliorer les soins aux personnes des Premiers Peuples et à d'autres personnes marginalisées, la formation en sciences infirmières ne devrait-elle pas se centrer sur le développement d'une conscience critique chez les apprenants, autant que sur le déploiement de 
leur jugement clinique infirmier (Beauchamp et al.; Lavoie et al., dans ce numéro de Avancées en formation infirmière [AFI]), de l'éthique et d'autres compétences prioritaires? Il est intéressant de noter que O'Flynn-Magee et al., rapportent, dans ce numéro de AFI, avoir été inspirés par les notions de pouvoir, oppression et agentivité humaine définies par Freire, pour la conception de leur atelier Cognitive Rehearsal to Address Bullying (CRAB) comprenant des activités participatives d'apprentissage visant à encourager un changement de perspective en situations d'intimidation. De plus, il est capital qu'une attention soit portée sur la meilleure approche possible pour favoriser le développement de la conscience critique chez les apprenants.

Des exemples peuvent être tirés de l'intervention éducative San'yas portant sur la sécurité culturelle pour les personnes Autochtones, une formation qui devient de plus en plus connue, à juste titre. Cette formation dirigée par des Autochtones, orientée vers les politiques et se situant au plan des systèmes «a pour buts de favoriser l'équité en santé et de réduire les formes interconnectées de racisme interpersonnel et structurel vécues par les personnes des Premiers Peuples dans le système de santé au Canada » (traduction libre, Browne et al., 2021, p.1). Les caractéristiques de ce programme indiquent un enseignement spécifique au sujet des Premiers Peuples, fait par eux, une approche qui vise à transformer les pensées et les actions et une intervention au plan des systèmes, puisque la formation seule ne pourrait se traduire en un changement organisationnel systémique des politiques (Browne et al., 2021).

Afin de continuer cette conversation sur l'équité en santé et dans les soins pour les Premiers Peuples, nous sommes honorées de souhaiter la bienvenue aux $\mathrm{D}^{\text {re }}$ Lisa B. Bearskin, $\mathrm{D}^{\text {re }}$ Andrea Kennedy, et $\mathrm{D}^{\text {re }}$ Wanda Phillips-Beck comme Éditrices invitées pour une prochaine édition spéciale de AFI. Restez à l'affut!

Jacinthe Pepin, RN PhD, Co-Editor-in-Chief - Corédactrice en chef

Florence Myrick, RN PhD, Co-Editor-in-Chief - Corédactrice en chef

\section{Références}

Association canadienne des écoles de sciences infirmières. (2015). National Nursing Education Framework. Final report. Auteur. https://www.casn.ca/wpcontent/uploads/2014/12/Framwork-FINAL-SB-Nov-30-20151.pdf

Association canadienne des écoles de sciences infirmières. (2021, 29 juin). Canadian Association of Schools of Nursing Statement of Commitment Anti-Indigenous Racism. https://www.casn.ca/wp-content/uploads/2021/07/CASN-Anti-Indigenous-Statement-ofCommitment-EN.pdf

Browne, A., Varcoe, C., et Ward, C. (2021). San'yas Indigenous Cultural Safety Training as an educational intervention: Promoting anti-racism and equity in health systems, policies, and practices. The International Indigenous Policy Journal, 12(3). https://doi.org/10.18584/iipj.2021.12.3.8204

Frank, J. R., Snell, L., et Sherbino, J. (dir.) (2015). CanMEDS 2015 Physician Competency Framework. Royal College of Physicians and Surgeons of Canada. 
http://canmeds.royalcollege.ca/uploads/en/framework/CanMEDS\%202015\%20Framewor k_EN_Reduced.pdf

Frenk, J., Chen, L., Bhutta, Z. A., Cohen, J., Crisp, N., Evans, T., Fineberg, H., Garcia, P., Yang, K., Kelley, P., Kistnasamy, B., Meleis, A., Naylor, D., Pablos-Mendez, A., Reddy, S., Scrimshaw, S., Sepulveda, J., Serwadda, D., et Zurayk, H. (2010). Health professionals for a new century: Transforming education to strengthen health systems in an interdependent world. The Lancet, 376(9756), 1923-1958. https://doi.org/10.1016/s0140$\underline{6736(10) 61854-5}$

Lavoie, P., et Deschênes, M-F. (sous presse). Fostering the development of clinical judgment and reasoning in the clinical setting. Dans S. Melrose et B. Perry (dir.), Clinical Teaching in Canadian Nursing. Canadian Association of Schools of Nursing.

Monteiro, S., Sherbino, J., Sibbald, M., et Norman, G. (2020). Critical thinking, biases and dual processing: The enduring myth of generalisable skills. Medical Education, 54(1), 66-73. https://doi.org/10.1111/medu.13872 